\title{
NUEVOS TIEMPOS, NUEVOS DESAFÍOS: GOBIERNO CORPORATIVO, UNA NUEVA ARISTA EN LA CREACIÓN DE VALOR'
}

\section{PABLO SAN MARTÍN MOSQUEIRA}

- Facultad de Ciencias Económicas y Administrativas, Universidad Católica de la Santísima Concepción

\section{ORLANDO LLANOS CONTRERAS}

- Facultad de Ciencias Económicas y Administrativas, Universidad Católica de la Santísima Concepción

\section{RESUMEN}

La importancia del buen gobierno corporativo ha comenzado a replantear la forma de desarrollar el quehacer económico de la empresa. Hasta hace no muchos años atrás era un concepto desconocido, hoy cada empresa que piensa con seriedad en su futuro, se plantea la idea de avanzar en temas de gobierno corporativo.

Por medio de este artículo se pretende reforzar la idea de que la empresa dispone de una serie de mecanismos de gobierno corporativo, tales como: a) la estructura de propiedad; b) los contratos de incentivos; c) el directorio; d) el endeudamiento; e) la transparencia en la contabilidad y f) el mercado de control corporativo, por medio de los cuales se puede, efectivamente, crear valor.

Palabras clave: Gobierno corporativo, Pequeña y mediana empresa

\section{ABSTRACT}

The importance of good corporate governance has taken us to rethink how to develop the economic affairs of the firm. Until not many years ago corporate governance was a rather unknown concept. Today, however, any firm that thinks seriously about its future, does ponder on matters of corporate governance. The objective of this article is to reinforce the idea that the firm has within a series of corporate governance mechanisms, such as ownership structure, incentive contracts, the board of directors, borrowing, accounting transparency, and the corporate control market, in terms of which it can effectively create value.

Key words: Corporate governance, Small and medium enterprises

1. UPC Review of Global Management, Volumen 3, Número 1, junio 2017
Desde hace ya algunos años hemos venido escuchando sobre el concepto de gobierno corporativo. Hasta hace poco era tema de conversación (y aparentemente de preocupación) solo de las grandes empresas. No obstante, una cantidad no menor de investigaciones científicas dejan ver la importancia de buenas prácticas de gobierno corporativo también en empresas de menor tamaño, además de la influencia que ello tiene sobre la creación de valor y la rentabilidad de la empresa.

En estricto rigor, esta literatura financiera se ha encargado de demostrar que la forma en que las compañías estructuran su gobierno tiene influencia sobre su capacidad competitiva, y, por ello, no son indiferentes las estructuras o el modo de gobierno que una empresa defina.

Volviendo al origen, al concepto de gobierno corporativo, no existe una única y amplia definición. La literatura teórica ofrece muchas, con diferentes enfoques. Sin embargo, todas ellas están construidas sobre la base de dos pilares fundamentales (Claessens y Yurtoglu, 2013). En cuanto al primero, existe un bloque de definiciones que lo vincula con un conjunto de patrones de comportamiento que reflejan decisiones financieras y de gestión. En segundo lugar, otro grupo de definiciones lo asocian con un marco normativo que, por tanto, define la forma en que las empresas se rigen. Aunando ambas visiones, podemos entender el gobierno corporativo, entonces, como el conjunto de mecanismos generados tanto interna como externamente (por ejemplo, normas, reglas, procedimientos, políticas e instituciones) a través del cual las empresas desarrollan su quehacer para poder alcanzar su objeto empresarial. Lo anterior, dado particularmente en un contexto en que existe separación entre la propiedad y la gestión de una compañía.

Ahora bien, es necesario destacar ciertos elementos en la definición. Primero, las empresas existen porque persiguen un objetivo de negocio. Segundo, el hecho de que quienes las gestionan sean personas distintas y contratadas por los dueños de la firma puede hacer que el objetivo empresarial no se cumpla a cabalidad. Tercero, 
debe establecerse una serie de mecanismos internos y externos que aseguren que el objetivo empresarial se lleve adelante del mejor modo posible.

Los mercados financieros en los que las compañías navegan sufren constantes cambios. Por ello es que, desde la mirada de todos los participantes, se hace indispensable reflexionar, analizar y proponer medidas para adoptar y con ello mejorar el gobierno corporativo. Así pues, desde hace pocos años escuchamos en el concierto internacional lo que se conoce como buenas prácticas de gobierno corporativo. En definitiva, una serie de recomendaciones emanadas desde distintas entidades internacionales que buscan orientar acerca de qué se espera que las empresas hagan en términos del buen gobierno de las mismas. Estas iniciativas dejan en evidencia que se está abogando por el buen gobierno de las empresas y por alcanzar esta meta realizando coordinaciones a diferentes niveles.

Con relación a con nuestra definición de gobierno corporativo, estas iniciativas, "buenas prácticas de gobierno corporativo", en términos concretos, tienen dos objetivos. Primero, realizar recomendaciones de alcance empresarial acerca de los mecanismos que estas debiesen establecer (mecanismos internos). Segundo, proponer a los países leyes, normativas e instituciones (mecanismos externos) que favorezcan el buen gobierno de las compañías.

Analizado desde el punto de visto empresarial, tiene lógica hacer del buen gobierno una práctica habitual; en definitiva, una organización bien gobernada es más valiosa y más rentable que una con menor calidad de gobierno, o al menos así lo demuestra la evidencia internacional (Chari et al., 2010; Klapper y Love, 2004; Love, 2011). ¿Pero qué interés podría tener un país en que sus empresas muestren mejor gobierno? La respuesta es simple: si empresas mejor gobernadas poseen mayor valor y mejor rendimiento, entonces aquel conjunto de empresas mejor gobernadas hará que el crecimiento del país se vea afectado positivamente. Y mayor crecimiento es sinónimo de mejores salarios, mayor empleo y, en consecuencia, mejor calidad de vida de los habitantes de ese país.

A modo de ejemplo, traemos a colación un escándalo corporativo vivido en Chile en el que se vio envuelta La Polar ${ }^{2}$ durante el año 2011. Una empresa que aparentemente lo estaba haciendo todo bien, hasta que se descubrió que existía en su interior un sistema confabulado para enriquecer de manera indebida a unos pocos. Claramente, podemos establecer que se trata de un problema de gobierno corporativo. También sabemos que las consecuencias de este caso salpicaron no solo a los propios trabajadores de la organización, a los accionistas minoritarios y a muchos clientes, sino también a todos quienes colocan dinero en las AFP ${ }^{3}$. Las AFP tenían,

\section{Empresa del sector Retail que opera en Chile.}

3. Se entiende por AFP a las Administradoras de Fondos de Pensiones, a la cuales el trabajador chileno se encuentra obligado a enviar el 10\% de su remuneración mensual, dinero que administran estas entidades privadas y que invierten en las mejores oportunidades de inversión (de acuerdo a lo que la ley chilena les permite), a efectos de rentabilizar aquellos recursos y con ello hacer crecer la futura pensión del trabajador. cual más cual menos, cantidades importantes de dinero invertidas en acciones de esta empresa. Acciones que de un día para otro comenzaron a perder su valor. Ello implica que los fondos para la futura pensión de los chilenos, invertidos en La Polar, también perdieron valor. Por lo tanto, ¿les interesan las compañías bien gobernadas a los países? La respuesta es, de modo evidente, sí. Empresas mejor gobernadas contribuyen al crecimiento de una nación. Empresas mal gobernadas destruyen valor para la economía.

\section{LA CREACIÓN DE VALOR}

En la sección anterior dejamos cimentadas las bases para poder continuar construyendo. Hablamos de una definición de gobierno corporativo, así como de la importancia que este tiene en la consecución del principal objetivo empresarial, que es la creación de valor. En esta sección, se busca seguir con el desarrollo de nuevos andamiajes que contribuyan al desarrollo de la idea de gobierno corporativo como forma de creación de valor para las empresas.

Primero que todo, es necesario reconocer las condiciones sobre las cuales las empresas latinoamericanas operan. Se indicó anteriormente que los mecanismos de gobierno corporativo son tanto internos como externos a la empresa. Si quisiéramos dimensionar el alcance del gobierno corporativo, podríamos pensar en una gráfica bidimensional, cuyo eje $X$ fuese la normativa legal imperante y las instituciones que la sostienen (lo que serían los mecanismos externos a la empresa), y en el eje Y estarían todas aquellas acciones generadas en el interior de cada empresa (mecanismos internos de gobierno corporativo). Respecto de lo primero, la dimensión horizontal, que entendemos como el piso sobre el cual las organizaciones operan, la evidencia internacional deja de manifiesto que el conjunto de países latinoamericanos tienen mucho aún por mejorar, y por ello se sitúan en la parte baja de las escalas que suelen utilizarse para medir calidad del entorno legal e institucional de una economía (Claessens et al., 2002; Morey et al., 2009). En este sentido, entonces, los países latinoamericanos deben seguir avanzando, y nuestra clase política ha de apurar el paso para mejorar los estándares en términos de transparencia, acceso y calidad de la información, y protección al inversionista, así como de la inclusión en nuestras economías de lo que se conoce como white paper, que no es otra cosa que un compendio de sugerencias para implementar buenas prácticas de gobierno en el interior de las empresas, un código de buen gobierno.

Todo lo anterior, el marco legal e institucional del país, no representa más que la base en común sobre la cual operan las empresas. Cuando el marco institucional mejora, lo hace para todos de manera conjunta. Y, como es sabido, todas las reformas estructurales, que modifican la base de operación de las empresas, dependen de la voluntad política que les impriman los Estados a la temática. No obstante, existe un conjunto de reformas internas que las organizaciones pueden desarrollar

Se ha dicho que la inversión en gobierno corporativo es rentable, y la evidencia internacional da cuenta de ello tanto para países desarrollados como para los que se encuentran en desarrollo (Lins, 2003; Love, 2011). Dicho en 
términos financieros, la inversión en gobierno corporativo tiene VAN (valor actual neto) positivo.

Qué hacer para mejorar la calidad del gobierno de la empresa. Citando a Michael Porter y Mark Kramer (2006) es necesario que las empresas incorporen dentro de su estrategia de negocio lo que la sociedad espera de ellas. No por cumplir ni limpiar su imagen, sino a efectos de incorporar las oportunidades que la propia sociedad les ofrece. Adicionalmente, la idea de gobierno corporativo no se centra en quién o quiénes ostentan el control de una compañía, sino en la manera de buscar la forma de cómo generar más valor y hacerse viable en el tiempo. Por esto es que se exponen en este párrafo ciertas orientaciones de largo plazo que ponen énfasis más allá de la última línea del estado de resultados del año que se haya cursado. En empresas de mediano tamaño y grandes, en las que existen, por una parte, los propietarios, y por otra, los que realizan la gestión, se espera la existencia de un directorio bien organizado, que represente efectivamente los intereses de los dueños y que sea capaz de visualizar, y con ello concretar, estrategias de largo plazo para la empresa. En términos específicos, se desea del directorio lo siguiente: primero, establecer y revisar las estrategias y políticas generales de la empresa, su forma de organización y funcionamiento; segundo, implementar un adecuado sistema de control de la información, en flujos tanto internos como externos; tercero, formalizar planes y métodos que ayuden a asegurar la continuidad de la organización en el tiempo (en empresas familiares, ayuda a establecer los protocolos familiares, las estrategias y los planes de sucesión de una generación a otra); cuarto, determinar estándares de comportamiento en el interior de la empresa, que podrían concretarse por medio del establecimiento de códigos de ética; quinto, definir políticas adecuadas de remuneración e incentivos, tanto para la alta gerencia como para los empleados; $y$, finalmente, pero no menos importante, supervisar a la alta gerencia en la correcta implementación de las políticas fijadas y en la forma en que desarrollan sus funciones, de modo que se alcance el objetivo empresarial.

Ahora bien, ¿qué ejemplo podemos seguir? ¿Qué patrones de conducta imitar? ¿Qué patrones de conducta inhibir? En lo que sigue se intenta, por medio de casos y ejemplos concretos, sugerir aquellos mejores mecanismos que ayuden al buen gobierno, a la optimización de la gestión, para lograr efectos positivos sobre el resultado y el valor de la empresa

\section{MECANISMOS Y HERRAMIENTAS QUE CONTRIBUYEN AL BUEN GOBIERNO}

Se ha destacado cómo, a la luz de investigaciones científicas, el buen gobierno de una empresa se traduce en mejores resultados para la misma. Aun así, el día a día muchas veces impide visualizar el largo plazo y percibir claramente el mejor camino por el cual la organización debe transitar hacia un mejor futuro. El foco en el corto plazo inhibe, en algunas circunstancias, la importancia que tienen aquellos elementos que pueden asegurar ese mejor futuro, es decir, el foco de largo plazo. Adicionalmente, modificaciones a la estructura de la empresa implican un costo, costo que ella no siempre estará dispuesta a asumir. Una vez llegados a este punto, cuando se ha atravesado el umbral y se reconoce la necesidad de avanzar en inversiones en gobierno corporativo, podemos dar cuenta de aquellas herramientas que contribuyen a una mejor forma de gobernar nuestro negocio.

Entre los mecanismos de gobierno corporativo de los que disponen los propietarios de una empresa se pueden mencionar los siguientes: a) la estructura de propiedad; b) los contratos de incentivos; c) el directorio; d) el endeudamiento; e) la transparencia en la contabilidad; y f) el mercado de control corporativo.

El primer mecanismo, la estructura de propiedad, hace referencia principalmente a que, mientras más concentrada se encuentre la propiedad (es decir, mientras menos sean los dueños de la empresa), entonces mayores incentivos tendrán para ejecutar acciones de supervisión y control sobre el actuar de la gerencia. Por tanto, mayor proporción de la propiedad de la empresa representa mayor interés por que a la compañía le vaya bien, por lo que se realizará el máximo esfuerzo en supervisión y control. Del mismo modo, también se espera que haya un alto interés en contratar a gestores que se alínean con las ideas de los propietarios, o en despedir a los que no lo hacen. El caso extremo se da cuando hay un solo dueño: aquí, los intereses son máximos, no existen responsabilidades compartidas y, regularmente, este único dueño actúa también como gestor de la empresa. Por otra parte, propietarios con disímiles porcentajes de propiedad podrían acarrear otro tipo de problemas. Los principales dueños supervisan eficientemente el actuar de los gestores, pero puede que en algunos casos prefieran buscar su propio beneficio, lo que podría perjudicar a propietarios menos importantes y sin capacidad de decisión.

Dos ejemplos extremos nos ayudan a comprender la importancia de la estructura de propiedad. La forma de presentación de estos ejemplos se efectúa por contraste, es decir, que los errores cometidos por otros sean situaciones que se puedan evitar en toda organización. Primero, la empresa chilena La Polar, con muchos dueños, pero ninguno de ellos con un porcentaje tal que represente motivación o interés por ejercer supervisión de manera efectiva, facilitó que la plana gerencial emprendiera una serie de iniciativas fraudulentas durante años, con el propósito de buscar su propio enriquecimiento de manera irregular. Segundo, el grupo económico Soquimich, uno de los conglomerados más grandes del mundo en producción de químicos no metálicos, que se encuentra societariamente estructurado de manera piramidal, tenía un accionista con un alto porcentaje de propiedad y otros muchos accionistas con porcentajes de propiedad pequeños, entre ellos algunas AFP. El controlador, abusando de su posición dominante, realizó actividades en su propio beneficio más que en el de la empresa, y mucho menos que en el de los accionistas pequeños.

Un segundo mecanismo, igualmente relevante, está representado por todas aquellas maneras de compensar a la plana gerencial. ¿Cuál es la mejor forma de 
compensación? ¿Sueldo fijo? No, debe haber motivación extra. ¿Sueldo únicamente variable? No, demasiada inestabilidad. El mix entre un monto fijo, que sea atractivo para una posición gerencial, más una parte variable en función del desempeño de la empresa, pareciera otorgar estabilidad y también desafío al gestor. La parte variable debe estar, no obstante, bien estudiada. Hablando claramente, una recompensa sobre el desempeño contable de la empresa pareciera ser una forma de incentivo inadecuada, puesto que abre la puerta a la potencial manipulación de la contabilidad. Por el contrario, muchas compañías han optado por recompensar por medio de acciones de la propia empresa, es decir, recompensar otorgando pequeñas porciones de la propiedad de la misma. Ello conlleva grandes ventajas: primero, aleja la posibilidad de manipular la contabilidad; segundo, el gestor, al ser parte también de la propiedad, tiende a alinear su comportamiento, pensando en el desarrollo de su función no solo como gerente, sino también como dueño.

Como tercer mecanismo se menciona el endeudamiento corporativo. El que la empresa haga uso del endeudamiento como forma de financiar sus actividades ayuda a que la plana gerencial tenga mayor disciplina. Los gerentes saben que deben trabajar periodo a periodo, fuertemente, en conseguir los recursos para hacer frente a las obligaciones financieras de la compañía. Conocen el monto del compromiso financiero y saben que deben tener el dinero suficiente para afrontarlo. Esta disciplina impone, regularmente, mayor rigurosidad y esfuerzo a la gerencia, puesto que no cumplir con los compromisos de deuda podría ocasionar consecuencias graves para la empresa. Y para ellos mismos, los gerentes, la pérdida de su fuente laboral.

El cuarto mecanismo que hemos mencionado es el directorio corporativo, que es un órgano de gobierno que vincula a los propietarios de la compañía con los gestores de la misma. Como sabemos, estos son los representantes de los propietarios y la plana gerencial les rinde cuentas y les presenta iniciativas, a las que el directorio da su visto bueno. En este entendido, el directorio es un elemento crucial para el buen gobierno de la empresa, así como para un buen rendimiento. La investigación acerca de este órgano de gobierno nos deja evidencias de que, mientras mayor proporción de directores independientes de los dueños controladores existan, mejor rendimiento experimentará la organización (Lefort y Urzúa, 2008). Lo anterior quiere decir que, cuanto mayor sea el número de directores que existan que no hayan sido elegidos o designados por los dueños mayoritarios de la empresa, mayor independencia tendrán para tomar decisiones en el interior de ella que beneficien a todos y no solo a unos pocos. Es decir, son directores que ostentan la independencia para actuar y decidir sobre la base de lo que es mejor para el futuro de la empresa y para todos sus dueños.

Un estudio de McKinsey e Icare, efectuado para Chile (McKinsey, 2007), pone de manifiesto que es justamente en el funcionamiento de los directorios donde más se necesita avanzar en materia de gobierno corporativo. Así, por ejemplo, el directorio de la empresa debería invertir el tiempo que sea preciso en el diseño de la estrategia de crecimiento para los próximos años y, de manera contraria, gastar poco tiempo en revisar los resultados pasados. En otros términos, su foco debe estar en el futuro más que en la historia de la empresa. Esto, a pesar de parecer un ejemplo en extremo simple, resulta ser exactamente lo inverso a lo que ocurre en la realidad. Los directores se enfrascan en revisar y debatir respecto de los resultados pasados, en vez de dedicarse a diseñar y planificar el crecimiento futuro de la compañía.

El quinto mecanismo, transparencia en la contabilidad, representa un gran desafío no solo para la organización, sino también para los legisladores. Si tomamos en consideración que los grandes escándalos financieros tuvieron su génesis en fraudes contables, podemos percibir que mejorar la transparencia de los sistemas contables, así como de la información que se provee, tiene mucha relevancia. La Polar, en el caso de Chile, o empresas como Enron o Parmalat, de modo internacional, dan cuenta de lo que se viene planteando. Mayor transparencia de la información ayuda a disminuir las brechas informativas de todos los actores relevantes en torno a la empresa (propietarios, directorio, gerencia, empleados y potenciales inversionistas, así como entidades fiscalizadoras).

Como sexto mecanismo, y último en esta serie, se comenta sobre el mercado de tomas de control. Supongamos que una empresa se encuentra en un mercado con alto potencial, pero ha venido evidenciando un mal desempeño financiero como consecuencia de algunas malas prácticas de gestión. Sumado a ello, su directorio no ha sido capaz de controlar la situación. Todo esto podría motivar que alguien fuera de la compañía (un inversionista u otra empresa, e incluso la competencia) prepare una oferta para hacerse con la compañía, comprarla. Las empresas siempre son escrutadas por diferentes actores de la economía. Un inversionista o la competencia podrían pensar que aquella empresa, en sus manos, tal vez tendría un mejor desempeño. Así, luego de un periodo de análisis y valoración, cuando ya están decididos, lanzan una oferta de compra, lo que tiene como resultado varias cosas. Primero, que el directorio de la compañía deja de ejercer sus funciones, puesto que son ahora los nuevos dueños quienes deberán elegir un nuevo directorio. Segundo, la plana gerencial es removida y, en su reemplazo, llega un nuevo equipo de confianza de los nuevos dueños que, se espera, sea capaz de sacar el máximo potencial posible de los negocios adquiridos, algo que ni el directorio anterior ni la plana gerencial lograron. El mercado de control corporativo, como mecanismo de gobierno corporativo, si bien es cierto que no es demasiado activo en nuestros países, reviste un real incentivo para quienes dirigen y gestionan una empresa, puesto que les obliga a disciplinarse a efectos de beneficiarla.

Concluimos entonces este diálogo, en el que hemos ahondado en temas de gobierno corporativo, su importancia para la compañía actual y algunos de los mecanismos al servicio de los propietarios para alcanzar un mejor gobierno de la empresa. 


\section{REFERENCIAS}

Chari, A., Ouimet, P. P. \& Tesar, L. L. (2010). The Value of Control in Emerging Markets. Review of Financial Studies, 23, 1741-1770.

Claessens, S., Djankov, S., Fan, J. P. H. \& Lang, L .H. P. (2002) Disentangling the Incentive and Entrenchment Effects of Large Shareholdings. The Journal of Finance, 57(6), 2741-2771.

Claessens, S. \& Yurtoglu, B. B. (2013). Corporate Governance in Emerging Markets: A Survey. Emerging Markets Review, 15, 1-33.

Klapper, L. F. \& Love, I. (2004). Corporate Governance, Investor Protection, and Performance in Emerging Markets. Journal of Corporate Finance, 10, 703-728.

Lefort, F. \& Urzúa, F. (2008). Board Independence, Firm Performance and Ownership Concentration: Evidence from Chile. Journal of Business Research, 61(6), 615-622.

Lins, K. V. (2003). Equity Ownership and Firm Value in Emerging Markets. The Journal of Financial and Quantitative Analysis, 38(1), 159-184

Love, I. (2011). Corporate Governance and Performance around the World: What We Know and What We Don't. World Bank Research Observer, 26(1), 42-70.

McKinsey \& Company. (2007). Documento de trabajo.

Morey, M., Gottesman, A., Baker, E. \& Godridge, B. (2009). Does Better Corporate Governance Result in Higher Valuations in Emerging Markets? Another Examination Using a New Data Set. Journal of Banking \& Finance, 33, 254-262.

Porter, M., \& Kramer, M. (Diciembre de 2006). Estrategia y Sociedad. Harvard Business Review. 